\title{
ON A NONLINEAR PROBLEM OF THE THEORY OF POTENTIAL FLOWS*
}

BY

\author{
ELIAS WEGERT
}

Bergakademie Freiberg

1. Introduction. In [2], N. Geffen considered some nonlinear boundary-value problems for harmonic functions which are related to problems of hydrodynamics. There, in particular, the following question is discussed.

Let $D$ be a two-dimensional simply-connected domain, the boundary $\partial D$ of which is decomposed into two parts, $\partial D_{1}$ and $\partial D_{2}$. We are looking for a potential flow with velocity field $V=(u, v)$ in $D$ whose absolute value $|V|=\left(u^{2}+v^{2}\right)^{1 / 2}$ is a given function $f(t)$ on $\partial D_{1}$ and whose normal component $V_{n}$ is a prescribed function $g(t)$ on $\partial D_{2}(t$ the arclength of the boundary).

Geffen conjectured that this problem has a solution at least for the case where

$$
\int_{\partial D_{2}} V_{n} d t=\int_{\partial D_{2}} g d t=0
$$

i.e., where there is no net flux through the boundary $\partial D_{2}$.

In the present paper we give an example which possesses no solution (in the class of functions which are square-summable on the boundary $\partial D$ ), although condition (1) is fulfilled.

2. Counterexample. We introduce the complex velocity $w=u-i v$, which is an analytic function in the domain $D$. Assume that $D$ is the complex unit disk $D=\{z=x+i y$ : $|z|<1\}$. Further, let $\partial D_{1}$ be the lower and $\partial D_{2}$ the upper half of the boundary $\partial D$ :

$$
\partial D_{1}=\{z=x+i y:|z|=1, y<0\}, \quad \partial D_{2}=\{z=x+i y:|z|=1, y>0\} .
$$

\footnotetext{
* Received June 26, 1986.
} 
The boundary conditions we are concerned with read as follows:

$$
\begin{array}{cl}
|V|^{2}=(u(t))^{2}+(v(t))^{2}=(f(t))^{2} & \text { on } \partial D_{1}, \\
V_{n}=u(t) \cos t+v(t) \sin t=g(t) & \text { on } \partial D_{2},
\end{array}
$$

where $t$ is the oriented angle between the point $z$ of $\partial D$ and the real axis $(0<t<\pi$ on $\partial D_{2}$ and $\pi<t<2 \pi$ on $\left.\partial D_{1}\right)$.

The conditions (2) and (3) give rise to a boundary-value problem for the analytic function $w=u-i v$, a so-called Riemann-Hilbert problem. One can find a survey of the theory of linear Riemann-Hilbert problems for instance in the monographs of N. I. Muskhelishvili [5], F. D. Gakhov [1], and E. Meister [4].

To get an example which has no solution choose, for instance, $f(t) \equiv \varepsilon, g(t)=\sin 2 t$, where $\varepsilon$ is a sufficiently small positive constant to be specified later. Notice that $g$ fulfils the hypothesis (1).

Assume that problem (2), (3) is solvable and $w_{0}=u_{0}-i v_{0}$ is a solution with a square-summable boundary function. Then replace the condition (2) by the linear relation

$$
u(t) \cos t+v(t) \sin t=h(t) \quad \text { on } \partial D_{1},
$$

where $h(t):=u_{0}(t) \cos t+v_{0}(t) \sin t$ is the normal component of the velocity of that solution $w_{0}$. To study the properties of the solution determine the velocity field from its normal components on the whole boundary given by (3) and (4). This Riemann-Hilbert problem is linear and its index equals -1 (in the sense of [1], [5]; cf. also [4], but in this notation we have $\kappa=-2$ ). Thus there is one necessary and sufficient condition which ensures the existence of a solution:

$$
\int_{0}^{\pi} g(t) d t+\int_{\pi}^{2 \pi} h(t) d t=0
$$

This means that the total flux through the boundary must be zero. Further, the solution is unique and can be given explicitly. We have

$$
\begin{aligned}
& u(t)=\cos t h_{1}(t)-\sin t H h_{1}(t), \\
& v(t)=\sin t h_{1}(t)+\cos t H h_{1}(t)
\end{aligned}
$$

on the boundary $\partial D$, where

$$
h_{1}(t):=\left\{\begin{array}{l}
g(t) \text { if } 0<t<\pi \\
h(t) \text { if } \pi<t<2 \pi
\end{array}\right.
$$

and $H$ denotes the singular integral operator of Hilbert type:

$$
H h_{1}(t)=\frac{1}{2 \pi} \int_{0}^{2 \pi} h_{1}(s) \cot \frac{s-t}{2} d s .
$$

From (5) we calculate the tangent component of the velocity field along the boundary:

$$
V_{t}=-\sin t u(t)+\cos t v(t)=H h_{1}(t) .
$$

Finally, we show that $V_{t}$ cannot be small near the boundary $\partial D_{1}$, which contradicts the estimate

$$
\left|V_{t}\right| \leqslant|V|=\varepsilon \text { on } \partial D_{1} .
$$


We have

$$
V_{t}(t)=H h_{1}(t)=\frac{1}{2 \pi} \int_{0}^{\pi} \sin 2 s \cot \frac{s-t}{2} d s+\frac{1}{2 \pi} \int_{\pi}^{2 \pi} h(s) \cot \frac{s-t}{2} d s .
$$

Since, by assumption, $|h(s)|=\left|V_{n}(s)\right| \leqslant \varepsilon$, on $\partial D_{1}$, the last integral is small; more precisely,

$$
\left\|\frac{1}{2 \pi} \int_{\pi}^{2 \pi} h(s) \cot \frac{s-t}{2} d s\right\|_{L_{2}(\pi, 2 \pi)} \leqslant\|h(s)\|_{L_{2}(\pi, 2 \pi)} \leqslant \varepsilon \pi^{1 / 2},
$$

where $\|\cdot\|_{L_{2}}$ denotes the norm of a function in the space $L_{2}$ of square-summable functions. To get the estimate (8) one must take into account that the norm of $H$ in $L_{2}(0,2 \pi)$ is equal to one and must extend the function $h$ to the interval $(0,2 \pi)$ by zero.

From (7) and (8) we conclude that

$$
\left\|V_{t}-V_{t}^{0}\right\|_{L_{2}(\pi, 2 \pi)} \leqslant \varepsilon \pi^{1 / 2}
$$

where the "main part" $V_{t}^{0}$ of $V_{t}$ is given by

$$
V_{t}^{0}(t)=\frac{1}{2 \pi} \int_{0}^{\pi} \sin 2 s \cot \frac{s-t}{2} d s
$$

We check that the function $V_{t}^{0}$ is bounded from below on $\partial D_{1}$ by a positive constant. We have

$$
\begin{gathered}
V_{t}^{0}(t)=\frac{1}{4 \pi} \int_{0}^{\pi / 2} \sin s\left(\cot \frac{s-2 t}{4}+\cot \frac{\pi-s-2 t}{4}-\cot \frac{\pi+s-2 t}{4}\right. \\
\left.-\cot \frac{2 \pi-s-2 t}{4}\right) d s .
\end{gathered}
$$

Since all the arguments of the cotangent functions belong to the interval $(-\pi, 0)$ whenever $0<s<\pi / 2$ and $\pi<t<2 \pi$, we get, after a simple consideration,

$$
\begin{array}{r}
\cot \frac{s-2 t}{4}-\cot \frac{\pi+2-2 t}{4} \geqslant 2 \tan \frac{\pi}{8}, \\
\cot \frac{\pi-s-2 t}{4}-\cot \frac{2 \pi-s-2 t}{4} \geqslant 2 \tan \frac{\pi}{8},
\end{array}
$$

and thus

$$
V_{t}^{0}(t) \geqslant \frac{1}{\pi} \tan \frac{\pi}{8}=c>0 \quad \text { if } \pi<t<2 \pi
$$

If we choose $\varepsilon<c / 2$, then (8), (9), and (10) lead to a contradiction:

$$
\varepsilon \pi^{1 / 2} \geqslant\left\|V_{t}-V_{t}^{0}\right\|_{L_{2}(\pi, 2 \pi)}>\|\varepsilon-2 \varepsilon\|_{L_{2}(\pi, 2 \pi)}=\varepsilon \pi^{1 / 2} .
$$

Hence a solution of (2), (3) cannot exist, provided that $\varepsilon$ is sufficiently small.

The physical meaning of this counterexample is the following. If on one part of the boundary $\partial D_{2}$ there is a "significant" flux of the fluid into the interior of $D$, and on the 
other part of $\partial D_{2}$ the fluid streams out of $D$ with equal flux, then it is impossible that the fluid is "nearly at rest" on $\partial D_{1}$.

3. Additional Remark. In [2], Geffen had reduced problem (2), (3) with $g(t) \equiv 0$ to some boundary-value problem for harmonic functions and had stated existence and uniqueness (in a sense) of the solution $w$. It is also possible to treat (2), (3) as a problem of Riemann-Hilbert type directly, as will be sketched here.

Following Geffen we assume that location and type of the stagnation points $z_{1}, \ldots, z_{n}$ of the flow are known a priori and factorize

$$
w(z)=\left(z-z_{1}\right)^{\beta_{1}} \cdots\left(z-z_{n}\right)^{\beta_{n}} w_{1}(z) .
$$

Now, the transformation $W=\log w_{1}$ leads to the problem

$$
\begin{aligned}
& \operatorname{Re} W=f_{1}(t) \quad \text { on } \partial D_{1}, \\
& \operatorname{Im} W=g_{1}(t) \quad \text { on } \partial D_{2},
\end{aligned}
$$

with given functions $f_{1}$ and $g_{1}$. This problem was examined by M. V. Keldysh and L. I. Sedov [3]. Moreover, they had studied the case where $\partial D_{1}$ and $\partial D_{2}$ consist of more than one connected component:

$$
\partial D_{1}=\partial D_{11} \cup \cdots \cup \partial D_{1 m}, \quad \partial D_{2}=\partial D_{21} \cup \cdots \cup \partial D_{2 m} .
$$

Note that, in general, the solution $W$ can be unbounded in a neighborhood of the points which are endpoints of both $\partial D_{1}$ and $\partial D_{2}$ if $m>1$. Keldysh and Sedov had stated that a (unique) bounded solution exists if and only if $m-1$ relations of the type

$$
\int_{\partial D_{1}} f_{1}(s) \lambda_{i}(s) d s+\int_{\partial D_{2}} g_{1}(s) \mu_{i}(s) d s=0, \quad i=1, \ldots, m-1,
$$

( $s$ the arclength on $\partial D$ ) are fulfilled. The real functions $\lambda_{i}$ and $\mu_{i}$ depend on the geometry of $D$ and on the partition of its boundary. For more details see also [1], Section 46.3.

\section{REFERENCES}

[1] F. D. Gakhov, Boundary value problems, Pergamon Press, Oxford, 1966

[2] N. Geffen, A nonstandard nonlinear boundary-value problem for harmonic functions, Quart. Appl. Math. 41, 289-300 (1983)

[3] M. V. Keldysh, L. I. Sedov, Effective solution of some boundary-value problems for harmonic functions, Dokl. Akad. Nauk SSSR (1) 16, 7-10 (1937)

[4] E. Meister, Randwertaufgaben der Funktionentheorie, B. G. Teubner, Stuttgart, 1983

[5] N. I. Muskhelishvili, Singular integral equations, P. Noordhoff N. V., Groningen, 1953 